\title{
Custo Total de Propriedade (TCO) aplicado para a Aquisição de um Refrigerador em uma Microempresa
}

\author{
Especialização em Gestão Estratégica de Pessoas pela Faculdade Educacional da \\ Andrei Moreira Neves
Faculdade Educacional da \\ Lapa - FAEL \\ Professor no Serviço Nacional de Aprendizagem Industrial - SENAI/SC \\ Rua Frei Bruno, 201 E. Parque das Palmeiras. Chapecó/SC. CEP 89.803-785 \\ E-mail: andreimoreiraneves@gmail.com
}

Daiane Izabele Ferreira de Souza

Graduação em andamento em Administração pela Universidade Federal da Fronteira

Sul - UFFS

Rodovia SC 484 - km 02. Bairro Fronteira Sul. Chapecó/SC. CEP: 89.815-899

E-mail: daiane.isabele@live.com

Moacir Francisco Deimling

Mestrado e Doutorado em Engenharia de Produção - UFRGS

Professor na Universidade Federal da Fronteira Sul - UFFS

Rodovia SC 484 - km 02. Bairro Fronteira Sul. Chapecó/SC. CEP: 89.815-899

E-mail: moacir.deimling@uffs.edu.br

\section{RESUMO}

O pequeno empresário possui dificuldade para a tomada de decisões nas empresas, principalmente quando se trata de aspectos financeiros. Esse desafio é ainda maior quando as decisões englobam custos ocultos. O presente artigo tem como objetivo calcular o custo total de aquisição de um equipamento refrigerador para suprir as necessidades apresentadas durante os eventos que são realizados em uma empresa de festas na cidade de Chapecó-SC. A pesquisa ocorreu no segundo semestre de 2017, e seu objetivo foi auxiliar os sócios da empresa na tomada de decisão, evidenciando o item mais viável para a organização. A pesquisa caracteriza-se como descritiva, com abordagem qualitativa do tipo aplicada, realizada por meio de um estudo de caso único. Em relação aos resultados, a ferramenta TCO permitiu verificar os custos totais, e o produto de menor valor de aquisição não se concretizou como a melhor opção de compra ao se analisar todos os custos envolvidos.

Palavras-chave: TCO. Custos. Custo total de propriedade. Gestão estratégica de custos. Ciclo de vida.

Total Cost of Ownership (TCO) applied for the acquisition of a Refrigerator in a Micro Enterprise 
Custo Total de Propriedade (TCO) aplicado para a Aquisição de um Refrigerador em uma Microempresa

Andrei Moreira Neves, Daiane Izabele Ferreira de Souza, Moacir Francisco Deimling

\section{ABSTRACT}

The small business owner has difficulty making decisions in companies, especially when it is related to economic aspects. This challenge is even greater when decisions involve hidden costs. This article aims to calculate or use the total refrigeration equipment to supply the needs during the events that are held in a party company in the city of Chapecó-SC. A survey took place in the second half of 2017 and its objective was to assist the company's business in decision making, highlighting the most viable item for the organization. A research described it as descriptive, with a qualitative approach of the applied type, carried out through a single case study. In relation to the results, the TCO of the tool allows to verify the maximum costs and the product with the lowest acquisition value does not materialize as the best purchase option to analyze all the costs involved.

Keywords: TCO. Costs. Total cost of ownership. Strategic cost management. Life cycle.

\section{INTRODUÇÃO}

As empresas, em um mercado cada vez mais competitivo, necessitam de boas decisões para que haja a sustentabilidade do negócio. As decisões estratégicas possuem reflexos nas operações da empresa, e isso é percebido pelos clientes. Quando se analisa um pequeno empreendimento, os investimentos são impactantes no orçamento da empresa e devem ser analisados com cautela.

A empresa em questão necessita de uma maior eficiência ao armazenar bebidas para serem consumidas nas festas. Atualmente, o processo ocorre com dificuldade e com o risco de as bebidas serem servidas em temperatura inadequada, ocasionando o descontentamento de seus clientes. Possuir um equipamento resfriador adequado, atendendo às especificações da empresa e também a sua realidade financeira é primordial para o resultado da organização, promovendo, assim, a competitividade e sustentabilidade da mesma.

Tendo isso em vista, busca-se responder ao seguinte problema de pesquisa: Qual a melhor opção de investimento para a aquisição de um refrigerador para uma empresa de festas em Chapecó-SC? 
Custo Total de Propriedade (TCO) aplicado para a Aquisição de um Refrigerador em uma Microempresa

Andrei Moreira Neves, Daiane Izabele Ferreira de Souza, Moacir Francisco Deimling

Tendo em vista as dificuldades com as quais a empresa vem se deparando, foi elaborado um artigo com a análise do custo total de propriedade para entender os custos englobados e adequá-los às necessidades da organização, para que, posteriormente, realizasse a aquisição de um refrigerador.

Portanto, foi realizada uma pesquisa descritiva com abordagem qualitativa, levantando os custos "ocultos" na aquisição, implementação, suporte e manutenção e o descarte ecologicamente de acordo com os métodos abordados no presente artigo.

Tem-se como objetivo geral: Analisar o TCO na aquisição de um equipamento refrigerador para uma empresa de festas em Chapecó-SC.

\section{REFERENCIAL TEÓRICO}

O conceito de Total Cost of Ownership ou, em tradução livre, Custo total de Propriedade (TCO), foi desenvolvido pelo Gartner Group, considerada uma das maiores empresas do mundo em consultoria e pesquisa do mercado de TI. A partir dos anos 1980, com a grande revolução tecnológica e o uso de computadores, havia a necessidade de se mensurar todos os custos envolvidos ao se adquirir esse equipamento (Cruz, 2017).

Conforme aborda o autor Cruz (2017), com o passar dos anos, a importância do custo total de propriedade cresceu na mesma proporção das tecnologias de informação e, com isso, os gestores necessitavam de ferramentas que apoiassem esse gerenciamento; assim, o TCO passou a ter uma relevância no apoio às decisões gerenciais.

O TCO é um cálculo que engloba todos os custos diretos e indiretos na aquisição de um bem. Não será calculado somente o custo de aquisição, mas todos os custos envolvidos durante o ciclo de vida do bem (Soutes, 2007). Para Ellram (2002), o TCO é uma técnica que apresenta os verdadeiros custos de um bem, possibilitando uma análise estratégica. 
Custo Total de Propriedade (TCO) aplicado para a Aquisição de um Refrigerador em uma Microempresa

Andrei Moreira Neves, Daiane Izabele Ferreira de Souza, Moacir Francisco Deimling

Cruz (2017) destaca que o TCO é uma métrica de análise com o objetivo de estimar os custos de vida e de aquisição. Esse método de avaliação oferece suporte à tomada de decisão. Borinelli (2003) aborda que o TCO é uma abordagem estruturada e essencial no processo de avaliação e seleção de fornecedores para uma adequada decisão de compra.

Para Camargo, Wernke, Zanin e Scheren (2016), as principais vantagens que o TCO oferece são: a identificação do valor total de um investimento em relação ao seu ciclo de vida; uma ferramenta para negociação e decisão devido à sua transparência; o encontro de custos "camuflados"; e a identificação da depreciação, da vida econômica e do ciclo de vida.

\subsection{Principais Custos Avaliados no TCO}

Deve-se ter uma compreensão acerca dos tipos de custos que são destacados na avaliação do TCO para que a empresa identifique os que estão presentes na sua realidade.

O custo de aquisição refere-se à compra, depreciação, compra de equipamentos auxiliares para compor a estrutura necessária e tempo gasto em horas de trabalho para pesquisa de mercado com relação à escolha de fornecedores. Já o custo de implementação diz respeito à contratação de consultores, que envolve configurações e instalação. Os custos de suporte e manutenção são contabilizados quando há implementação de novas funcionalidades, garantias, contratações de fornecedores externos e de recursos humanos (Cruz, 2017).

Os custos diretos possuem como característica principal a possibilidade de sua quantificação. Envolve principalmente aquisição e suporte. Os custos indiretos envolvem o suporte casual ou, ainda, atividades reparadoras ligadas à perda de produtividade, paradas e contratempos (Cruz, 2017).

Existem também os custos de descarte, que são aqueles relacionados ao envio do produto que se tornou inoportuno para uma destinação correta (Alves \& Cardoso, 2017). Coser e Souza (2017 apud Prabhakar \& Sandborn, 2012), apontam que "o 
Custo Total de Propriedade (TCO) aplicado para a Aquisição de um Refrigerador em uma Microempresa

Andrei Moreira Neves, Daiane Izabele Ferreira de Souza, Moacir Francisco Deimling

conceito de ciclo de vida concentra-se sobre o capital investido em ativos fixos, com ênfase em entender quanto realmente estes ativos custam para serem adquiridos, utilizados, mantidos e alienados ao final da sua vida útil".

Com o cálculo do Custo Total de Propriedade, é possível verificar os principais custos ligados à aquisição e uso, mas também é importante levar em conta as modalidades de vida útil relacionadas aos produtos e ativos que podem ser (Cruz, 2017):

A. Vida depreciável: o número de anos em que o ativo ou produto será depreciado.

B. Vida econômica: anos em que a aquisição consegue dar retorno financeiro para seu proprietário, que pode ser calculado a partir dos custos necessários para mantê-lo e operá-lo. Se tais custos excederem o seu retorno, já é um sinal de que sua vida econômica terminou.

Na Figura 1, são apresentadas as principais categorias de custos avaliadas pelo TCO segundo Coser e Souza (2017).

\begin{tabular}{|c|c|c|c|}
\hline $\begin{array}{l}\text { Categorias } \\
\text { de Custos }\end{array}$ & Autor/Ano & Descrição & Exemplos de Indicadores \\
\hline $\begin{array}{l}\text { Custo de } \\
\text { Iransação }\end{array}$ & $\begin{array}{l}\text { Ellram e Siferd } \\
\text { (1993): Weber } \\
\text { ef al. (2010). }\end{array}$ & $\begin{array}{l}\text { Atividades relacionadas à } \\
\text { seleção, } \\
\text { efetivação e manulificaçăono. } \\
\text { de fornecedores. Envolve } \\
\text { todos os custos burocráticos } \\
\text { associados a um pedido. }\end{array}$ & $\begin{array}{l}\text { Transação; Tempo; Atraso: } \\
\text { Garantia; Seguro; Projeto: } \\
\text { Taxas; Identificação de } \\
\text { necessidades: Pagamentos; } \\
\text { Consultas: Desenvolvimento; } \\
\text { Contatos: Despesas com } \\
\text { pessoal. }\end{array}$ \\
\hline $\begin{array}{l}\text { Custo de } \\
\text { Qualidade }\end{array}$ & $\begin{array}{l}\text { Gonen e Yukcu } \\
\text { (2012): Ferrin e } \\
\text { Plank (2002). }\end{array}$ & $\begin{array}{l}\text { Inclui os indicadores de } \\
\text { custos relacionados à } \\
\text { qualidade. Contempla os } \\
\text { custos de prevenção e os } \\
\text { custos de problemas de má } \\
\text { qualidade. } \\
\end{array}$ & $\begin{array}{l}\text { Planejamento e prevenção; } \\
\text { Avaliação de processos; } \\
\text { Custos de falhas internas; } \\
\text { Custos de falhas externas; } \\
\text { Controle e monitoramento; } \\
\text { Auditorias; Desperdicio. } \\
\end{array}$ \\
\hline $\begin{array}{l}\text { Custo do } \\
\text { Ciclo de } \\
\text { Vida }\end{array}$ & $\begin{array}{l}\text { Al-Alawi e } \\
\text { Bradley (2013): } \\
\text { Prabhakar e } \\
\text { Sandborn } \\
\text { (2012). }\end{array}$ & $\begin{array}{l}\text { Custos relacionados } \\
\text { diretamente à aquisição de } \\
\text { um produto, vistos no longo } \\
\text { prazo, ou seja, no ciclo de } \\
\text { vida do produto. }\end{array}$ & $\begin{array}{l}\text { Custo de aquisição, Custo de } \\
\text { empréstimos: Custo fiscal; } \\
\text { Descarte; } \\
\text { Frete; Ciclo de vida; } \\
\text { Obsolescência; Revenda; } \\
\text { Custos adicionais. }\end{array}$ \\
\hline
\end{tabular}

Figura 1. Principais categorias de custo do TCO

Fonte: Coser \& Souza, 2017. 
Custo Total de Propriedade (TCO) aplicado para a Aquisição de um Refrigerador em uma Microempresa

Andrei Moreira Neves, Daiane Izabele Ferreira de Souza, Moacir Francisco Deimling

Observa-se, no quadro acima, que os custos de transação são aqueles relacionados com os fornecedores, englobados no pedido de um bem. Os custos de qualidade são os de manutenção do bem, como, por exemplo, um seguro ou um conserto necessário. Os custos de ciclo de vida englobam todos os custos desde o momento em que se adquire um novo bem até o momento em que há o descarte.

\section{PROCEDIMENTOS METODOLÓGICOS}

A pesquisa classifica-se como descritiva e com abordagem qualitativa. Descritiva, pois, segundo Gil (2002), há um estabelecimento de relação das variáveis e servem para proporcionar uma visão do fenômeno e preocupam-se com uma atuação prática. Em uma pesquisa qualitativa, há um aprofundamento teórico em relação à situação abordada, buscando descrevê-la (Gerhardt \& Silveira, 2009). Godoy (1995) destaca que a pesquisa qualitativa tem preocupação fundamental com o estudo e a análise, proporcionando contato entre o pesquisador e com o ambiente em que a situação está sendo estudada. A pesquisa é do tipo aplicada, pois está direcionada a aplicar o seu resultado de forma real.

Este estudo foi desenvolvido por meio de um estudo de caso único. O estudo de caso, segundo Godoy (1995), caracteriza-se quando é analisado profundamente um ambiente ou uma situação em particular. A empresa de festas analisada é considerada uma microempresa conforme o Serviço Brasileiro de Apoio às Micro e Pequenas Empresas (2019), pois é um empreendimento que tem receita bruta anual inferior ou igual a $\mathrm{R} \$ 360$ mil. A empresa investigada possui três unidades na cidade de Chapecó, em Santa Catarina, e a presente investigação será aplicada na unidade dois, a qual possui espaço para buffet infantil e eventos. A unidade é composta por uma equipe de dois sócios e está em atividade desde o mês de janeiro de 2017. 
Custo Total de Propriedade (TCO) aplicado para a Aquisição de um Refrigerador em uma Microempresa Andrei Moreira Neves, Daiane Izabele Ferreira de Souza, Moacir Francisco Deimling

\section{RESULTADOS E DISCUSSÃO}

Neste tópico, primeiramente apresentar-se-á a empresa objeto do estudo e, posteriormente, o levantamento dos requisitos necessários para o equipamento a ser adquirido pela empresa e o processo de TCO.

\subsection{A Empresa e o Objeto de Estudo}

A empresa investigada é uma prestadora de serviços no setor de eventos. A unidade pesquisada possui, no seu quadro de funcionários, os seus dois sócios que executam todas as atividades da empresa.

Com o decorrer do desenvolvimento das atividades, houve a necessidade de mais um equipamento para refrigerar apenas as bebidas que serão servidas durante os eventos, pois, atualmente, as bebidas são refrigeradas juntamente com os demais alimentos que serão servidos na festa, restando apenas o freezer, quando não há mais espaço no refrigerador da empresa. Além disso, é utilizada uma geladeira doméstica que não atende adequadamente a necessidade do buffet.

Para suprir essa demanda, a empresa optou pela compra de um novo equipamento, visando a uma maior qualidade e segurança no resfriamento das bebidas que serão servidas aos seus clientes.

Visto a grande oferta no mercado e a variação de preços, ela necessita alocar seus recursos de forma a suprir sua necessidade e investir apenas o necessário. No atual contexto da empresa, visa-se identificar a opção com o menor custo total de propriedade.

\subsection{Levantamento de Requisitos e Processo do TCO}

Por meio de uma entrevista semiestruturada que permite perguntas centrais ao tema e a possibilidade de incluir novos questionamentos, conforme a necessidade do pesquisador (Gil, 2010) realizou-se o levantamento das necessidades da empresa e os requisitos que o equipamento deveria atender para suprir a atual e a futura 
Custo Total de Propriedade (TCO) aplicado para a Aquisição de um Refrigerador em uma Microempresa

Andrei Moreira Neves, Daiane Izabele Ferreira de Souza, Moacir Francisco Deimling

necessidade. O levantamento dos requisitos ocorreu no mês de outubro de 2017 , com a presença de ambos os sócios.

Mediante as necessidades identificadas na organização, os pesquisadores indagaram sobre quais seriam os requisitos mínimos que o equipamento deveria atender, e foram observados os seguintes critérios:

1. Capacidade para 150 litros;

2. Tensão $220 \mathrm{~V}$.

A partir do levantamento dos requisitos, segue-se para a construção do TCO. A operação deve ser seguida conforme o método exige para se atingir um resultado adequado. Com isso, Gasparetto e Silva (2004) destacam as atividades na operacionalização do TCO:

1. Atividades de aquisição;

2. Determinação dos custos mais relevantes;

3. Custeio das atividades envolvidas;

4. Alocação dos custos;

5. Indicadores de desempenho.

As cinco etapas são essenciais para se atingir o objetivo proposto e, com isso, inicia-se com as atividades de aquisição. Essas atividades dizem respeito principalmente ao levantamento de fornecedores, preços e às condições de entrega.

Realizou-se uma pesquisa no dia 01 de novembro de 2017, visando encontrar os modelos que se enquadram nos requisitos mínimos propostos, levantando os fornecedores e os preços. Esse levantamento pode ser observado na Tabela 1. 
Custo Total de Propriedade (TCO) aplicado para a Aquisição de um Refrigerador em uma Microempresa

Andrei Moreira Neves, Daiane Izabele Ferreira de Souza, Moacir Francisco Deimling

Tabela 1

Refrigeradores e seus respectivos valores

\begin{tabular}{|c|c|c|c|c|c|c|c|}
\hline \multicolumn{8}{|c|}{ Refrigerador } \\
\hline Marca & Modelo & $\begin{array}{c}\text { Capacidade } \\
\text { Armaz. (L) }\end{array}$ & Loja & Va & r a prazo & Va & or a vista \\
\hline \multirow{3}{*}{ Gelopar } & \multirow{3}{*}{$\begin{array}{l}\text { Gtpc-575 } \\
\text { (fechado) }\end{array}$} & \multirow{3}{*}{578} & Americanas & RS & $2.999,90$ & $\mathrm{R} \$$ & $2.849,91$ \\
\hline & & & Magazine Luiza & RS & $2.999,90$ & RS & $2.699,91$ \\
\hline & & & Ponto Frio & $\mathrm{RS}$ & $3.599,00$ & $\mathrm{RS}$ & $3.419,05$ \\
\hline \multirow{3}{*}{ Metalfrio } & \multirow{3}{*}{ Vb40r } & \multirow{3}{*}{406} & Americanas & $\mathrm{R} \$$ & $3.130,00$ & $\mathbf{R} \$$ & $2.973,50$ \\
\hline & & & Wallmart & $\mathrm{R} \$$ & $2.970,00$ & $\mathbf{R} \$$ & $2.970,00$ \\
\hline & & & Catral & $\mathrm{RS}$ & $3.237,39$ & $\mathrm{RS}$ & $2.913,65$ \\
\hline \multirow{3}{*}{ Metalfrio } & \multirow{3}{*}{ Vf56D } & \multirow{3}{*}{539} & Frigelar & RS & $3.299,00$ & RS & $3.068,07$ \\
\hline & & & Americanas & RS & $3.250,00$ & $\mathrm{RS}$ & $3.250,00$ \\
\hline & & & Submarino & RS & $3.250,00$ & RS & $3.250,00$ \\
\hline \multirow{3}{*}{ Gelopar } & \multirow{3}{*}{ Gptu-40 } & \multirow{3}{*}{414} & Americanas & $R \$$ & $2.769,00$ & $\mathbf{R} \$$ & $2.630,55$ \\
\hline & & & Magazine Luiza & $\mathrm{RS}$ & $3.299,00$ & RS & $2.969,10$ \\
\hline & & & Submarino & $\mathrm{R} \$$ & $2.769,00$ & $\mathbf{R} \$$ & $2.630,55$ \\
\hline \multirow{3}{*}{ Gelopar } & \multirow{3}{*}{ Gptu 230} & \multirow{3}{*}{156} & Submarino & RS & $3.877,08$ & RS & $3.683,23$ \\
\hline & & & Shoptime & $\mathrm{R} \$$ & $3.877,08$ & $\mathbf{R} \$$ & $3.683,23$ \\
\hline & & & Americanas & $\mathrm{RS}$ & $3.877,08$ & RS & $3.683,23$ \\
\hline \multirow{3}{*}{ Gelopar } & \multirow{3}{*}{ Gptu-230pr } & \multirow{3}{*}{228} & Shoptime & RS & $2.943,90$ & RS & $2.943,90$ \\
\hline & & & Ricardo Eletro & $R \$$ & $2.861,10$ & $R \$$ & $2.861,10$ \\
\hline & & & Magazine Luiza & $\mathbf{R} \$$ & $2.899,00$ & $\mathrm{R} \$$ & $2.609,10$ \\
\hline \multirow{2}{*}{ Metalfrio } & \multirow{2}{*}{ VB25R } & \multirow{2}{*}{235} & Carrefour & RS & $2.049,00$ & RS & $2.049,00$ \\
\hline & & & e-fácil & $\mathrm{RS}$ & $2.199,00$ & $\mathrm{RS}$ & $1.979,41$ \\
\hline
\end{tabular}

Nota. Fonte: Elaborada pelos autores.

A empresa realizaria o pagamento à vista por meio de boleto bancário. Então os custos financeiros não foram contabilizados. A segunda etapa descreve a determinação dos custos mais relevantes. Soutes (2007) destaca que o "custo total de propriedade tem sido definido como a soma de todos os custos e despesas associados com a compra e o uso de equipamentos, materiais e serviços".

As etapas três e quatro são realizadas simultaneamente. Por meio da identificação dos modelos de equipamentos que iriam cumprir as especificações, identificou-se o consumo de energia de cada um deles. Na Tabela 2, pode ser observada a tarifa aplicada sobre o consumo de energia, e a Tabela 3 apresenta o consumo de energia de cada refrigerador. 
Custo Total de Propriedade (TCO) aplicado para a Aquisição de um Refrigerador em uma Microempresa

Andrei Moreira Neves, Daiane Izabele Ferreira de Souza, Moacir Francisco Deimling

Tabela 2

Tarifas de consumo de energia

\begin{tabular}{ccc}
\hline \multicolumn{3}{c}{ Resolução homologatória no 2.286, de 15 de Agosto de 2017 } \\
\hline \multicolumn{1}{c}{ Tarifa convencional - Grupo B (sem tributos) } \\
\hline Classificação & Energia R\$/kWh \\
B1 & Residencial normal & 0,4598500 \\
& Residencial baixa renda até 30kWh & 0,1497265 \\
& Residencial baixa renda de 31 a 100kWh & 0,2566740
\end{tabular}

Nota. Fonte: CELESC, 2017.

Tabela 3

Consumo de energia de cada refrigerador

\begin{tabular}{|c|c|c|}
\hline Modelo & Consumo energia (mês) & $\begin{array}{c}\text { Valor de consumo de energia } \\
\text { (mês) }\end{array}$ \\
\hline Gtpc-575 & 65,1 & 29,936235 \\
\hline vb40r & 85 & 39,08725 \\
\hline vf56D & 105 & 48,28425 \\
\hline Gptu-40 & 126 & 57,9411 \\
\hline Gptu 230 & 42 & 19,3137 \\
\hline Gptu -230pr & 42 & 19,3137 \\
\hline VB25R & 185 & 85,07225 \\
\hline
\end{tabular}

Nota. Fonte: Elaborada pelos autores.

Com base nas informações coletadas, foram selecionados os fornecedores que apresentam o menor valor à vista para cada um dos modelos. De acordo com a M\&M Assessoria Contábil (2017), a depreciação anual de um bem como o refrigerador é de $10 \%$ ao ano. Essa informação da depreciação é baseada nas Instruções Normativas 
Custo Total de Propriedade (TCO) aplicado para a Aquisição de um Refrigerador em uma Microempresa

Andrei Moreira Neves, Daiane Izabele Ferreira de Souza, Moacir Francisco Deimling

SRF n. ${ }^{\text {s }} 162 / 98$ e $130 / 99$, conforme aponta M\&M. Com isso, pode-se calcular o custo de depreciação mensal, apresentada na Tabela 4.

Tabela 4

Custo de depreciação de cada modelo de refrigerador

\begin{tabular}{|c|c|c|}
\hline Modelo & Valor de aquisição & Custo de depreciação (mês) \\
\hline Gtpc-575 & $\mathrm{R} \$ 2.699,91$ & 22,49925 \\
\hline vb40r & $\mathrm{R} \$ 2.913,65$ & 24,28041667 \\
\hline vf56D & $\mathrm{R} \$ 3.068,07$ & 25,56725 \\
\hline Gptu-40 & $\mathrm{R} \$ 2.630,65$ & 21,92208333 \\
\hline Gptu 230 & $\mathrm{R} \$ 3.683,23$ & 30,69358333 \\
\hline Gptu -230pr & $\mathrm{R} \$ 2.609,10$ & 21,7425 \\
\hline VB25R & $\mathrm{R} \$ 1.979,41$ & 16,49508333 \\
\hline
\end{tabular}

Nota. Fonte: Elaborada pelos autores.

Tanto os custos de energia como o de depreciação são os custos de utilização (Alves \& Cardoso, 2017). Na Tabela 5, observa-se o custo mensal para manter o equipamento funcionando.

Tabela 5

Custo mensal para manter o refrigerador

\begin{tabular}{lcccc}
\hline Modelo & $\begin{array}{c}\text { Consumo } \\
\text { energia (mês) }\end{array}$ & $\begin{array}{c}\text { Valor de } \\
\text { consumo de } \\
\text { energia (mês) }\end{array}$ & $\begin{array}{c}\text { Custo de depreciação } \\
\text { (mês) }\end{array}$ & $\begin{array}{c}\text { Custo total mês } \\
\text { (energia+ depreciação) }\end{array}$ \\
\hline Gtpc-575 & 65,1 & 29,936235 & 22,49925 & $\mathrm{R} \$ 52,44$ \\
vb40r & 85 & 39,08725 & 24,28041667 & $\mathrm{R} \$ 63,37$ \\
vf56D & 105 & 48,28425 & 25,56725 & $\mathrm{R} \$ 73,85$ \\
Gptu-40 & 126 & 57,9411 & 21,92208333 & $\mathrm{R} \$ 79,86$ \\
Gptu 230 & 42 & 19,3137 & 30,69358333 & $\mathrm{R} \$ 50,01$ \\
Gptu -230pr & 42 & 19,3137 & 21,7425 & $\mathrm{R} \$ 41,06$ \\
VB25R & 185 & 85,07225 & 16,49508333 & $\mathrm{R} \$ 101,57$ \\
\hline
\end{tabular}

Nota. Fonte: Elaborada pelos autores. 
Custo Total de Propriedade (TCO) aplicado para a Aquisição de um Refrigerador em uma Microempresa

Andrei Moreira Neves, Daiane Izabele Ferreira de Souza, Moacir Francisco Deimling

Tendo o custo com energia mensal e o custo de depreciação mensal, é possível calcular o valor anual desses custos ao fim do ciclo de vida do equipamento. Os cálculos são apresentados na Tabela 6.

Tabela 6

Custo ao fim do ciclo de vida do equipamento

\begin{tabular}{lccc}
\hline Modelo & $\begin{array}{c}\text { Ciclo de vida } \\
\text { médio (anos) }\end{array}$ & $\begin{array}{c}\text { Custo de energia e } \\
\text { depreciação anual }\end{array}$ & $\begin{array}{c}\text { Custo de energia e } \\
\text { depreciação ao fim do ciclo } \\
\text { de vida }\end{array}$ \\
\hline Gtpc-575 & & $\mathrm{R} \$ 629,23$ & $\mathrm{R} \$ 6.292,26$ \\
vb40r & $\mathrm{R} \$ 760,41$ & $\mathrm{R} \$ 7.604,12$ \\
vf56D & $\mathrm{R} \$ 886,22$ & $\mathrm{R} \$ 8.862,18$ \\
Gptu-40 & 10 & $\mathrm{R} \$ 958,36$ & $\mathrm{R} \$ 9.583,58$ \\
Gptu 230 & & $\mathrm{R} \$ 600,09$ & $\mathrm{R} \$ 6.000,87$ \\
Gptu -230pr & $\mathrm{R} \$ 492,67$ & $\mathrm{R} \$ 4.926,74$ \\
VB25R & $\mathrm{R} \$ 1.218,81$ & $\mathrm{R} \$ 12.188,08$ \\
\hline
\end{tabular}

Nota. Fonte: Elaborada pelos autores.

Para chegar ao resultado do TCO, Soutes (2007), in: Riggs e Robbins (in: Bierma \& Waterstraat, 2004) sugerem uma fórmula para capturar todos os custos do produto. Ela é a seguinte: $\mathrm{TCO}=\mathrm{CM}+\mathrm{PC}+\mathrm{F}+\mathrm{M}+\mathrm{O}+\mathrm{CD}-\mathrm{VD}$ :

onde: $\mathrm{CM}=$ Custo dos Materiais secundários ou complementares; $\mathrm{PC}=$ Preço de Compra; $\mathrm{F}=$ Frete e transporte; $\mathrm{M}=$ Custos de manutenção; $\mathrm{O}=$ Custos de operação; $C D=$ Custos de Descarte; VD = Valor de Descarte.

Alves e Cardoso (2017) destacam que os custos mais relevantes são: transporte, instalação, propriedade, utilização, manutenção e descarte. O presente estudo baseouse na abordagem de Alves e Cardoso (2017), considerando a contribuição de Soutes (2007). Aplica-se então a fórmula: 
Custo Total de Propriedade (TCO) aplicado para a Aquisição de um Refrigerador em uma Microempresa

Andrei Moreira Neves, Daiane Izabele Ferreira de Souza, Moacir Francisco Deimling

$\mathrm{TCO}=$ Valor de aquisição + Custo de transporte + Custo de utilização + Custo de descarte.

A partir deste método, considerando-se que a vida útil estimada de um refrigerador que é de 10 anos e que se está desconsiderando, nesta análise, os possíveis custos com manutenção do refrigerador, por estes serem de difícil estimativa, chega-se à Tabela 7 do TCO.

Tabela 7

Custo total de propriedade

\begin{tabular}{lccccc}
\hline \multicolumn{1}{c}{ Modelo } & $\begin{array}{c}\text { Valor de } \\
\text { aquisição }\end{array}$ & $\begin{array}{c}\text { Custo de } \\
\text { transporte }\end{array}$ & $\begin{array}{c}\text { Custo de utilização } \\
\text { (Depreciação+ener- } \\
\text { gia) }\end{array}$ & $\begin{array}{c}\text { Custo de } \\
\text { descarte } \\
\text { (ecológico) }\end{array}$ & TCo \\
\hline Gtpc-575 & $\mathrm{R} \$ 2.699,91$ & $\mathrm{R} \$ 39,90$ & $\mathrm{R} \$ 6.292,26$ & $\mathrm{R} \$ 199,90$ & $\mathrm{R} \$ 9.231,97$ \\
vb40r & $\mathrm{R} \$ 2.913,65$ & $\mathrm{R} \$ 244,00$ & $\mathrm{R} \$ 7.604,12$ & $\mathrm{R} \$ 199,90$ & $\mathrm{R} \$ 10.961,67$ \\
vf56D & $\mathrm{R} \$ 3.068,07$ & $\mathrm{R} \$ 110,20$ & $\mathrm{R} \$ 8.862,18$ & $\mathrm{R} \$ 199,90$ & $\mathrm{R} \$ 12.240,35$ \\
Gptu-40 & $\mathrm{R} \$ 2.630,65$ & $\mathrm{R} \$ 176,00$ & $\mathrm{R} \$ 9.583,58$ & $\mathrm{R} \$ 199,90$ & $\mathrm{R} \$ 12.590,13$ \\
Gptu 230 & $\mathrm{R} \$ 3.683,23$ & $\mathrm{R} \$ 39,90$ & $\mathrm{R} \$ 6.000,87$ & $\mathrm{R} \$ 199,90$ & $\mathrm{R} \$ 9.923,90$ \\
Gptu -230pr & $\mathrm{R} \$ 2.609,10$ & $\mathrm{R} \$ 39,90$ & $\mathrm{R} \$ 4.926,74$ & $\mathrm{R} \$ 199,90$ & $\mathrm{R} \$ 7.775,64$ \\
VB25R & $\mathrm{R} \$ 1.979,41$ & $\mathrm{R} \$ 139,90$ & $\mathrm{R} \$ 12.188,08$ & $\mathrm{R} \$ 199,90$ & $\mathrm{R} \$ 14.507,29$ \\
\hline
\end{tabular}

Nota. Fonte: Elaborada pelos autores.

A empresa planeja descartar o equipamento ao fim de sua vida útil de forma adequada e, com base nas informações da empresa Ecycle, o valor para descarte correto deste equipamento será de $\mathrm{R} \$ 199,90$.

Na Tabela 8, visualiza-se o valor de aquisição, o TCO e os custos ocultos ao fim da vida útil do equipamento. Esses custos ocultos são os que normalmente não são avaliados pelas empresas, principalmente pelas pequenas. 
Custo Total de Propriedade (TCO) aplicado para a Aquisição de um Refrigerador em uma Microempresa

Andrei Moreira Neves, Daiane Izabele Ferreira de Souza, Moacir Francisco Deimling

Tabela 8

Custos ocultos

\begin{tabular}{lcccc}
\hline Modelo & $\begin{array}{c}\text { Capacidade } \\
\text { Armaz. (L) }\end{array}$ & Valor de aquisição & TCo & Custos ocultos \\
\hline Gtpc-575 & 578 & $\mathrm{R} \$ 2.699,91$ & $\mathrm{R} \$ 9.231,97$ & $\mathrm{R} \$ 6.532,06$ \\
vb40r & 406 & $\mathrm{R} \$ 2.913,65$ & $\mathrm{R} \$ 10.961,67$ & $\mathrm{R} \$ 8.048,02$ \\
vf56D & 539 & $\mathrm{R} \$ 3.068,07$ & $\mathrm{R} \$ 12.240,35$ & $\mathrm{R} \$ 9.172,28$ \\
Gptu-40 & 414 & $\mathrm{R} \$ 2.630,65$ & $\mathrm{R} \$ 12.590,13$ & $\mathrm{R} \$ 9.959,48$ \\
Gptu 230 & 156 & $\mathrm{R} \$ 3.683,23$ & $\mathrm{R} \$ 9.923,90$ & $\mathrm{R} \$ 6.240,67$ \\
Gptu -230pr & 228 & $\mathrm{R} \$ 2.609,10$ & $\mathrm{R} \$ 7.775,64$ & $\mathrm{R} \$ 5.166,54$ \\
VB25R & 235 & $\mathrm{R} \$ 1.979,41$ & $\mathrm{R} \$ 14.507,29$ & $\mathrm{R} \$ 12.527,88$ \\
\hline
\end{tabular}

Nota. Fonte: Elaborada pelos autores.

É possível destacar que o equipamento de menor valor possui o maior volume de custos ocultos e também o maior TCO. Esse dado é extremamente importante, visto que, se a empresa fosse analisar em qual dos equipamentos iria investir e levando em consideração apenas o valor de aquisição, acabaria pagando mais caro se fossem considerados todos os custos envolvidos.

Dentre os equipamentos analisados, as melhores opções para os sócios são os modelos Gptu - 230pr, com TCO R\$ 7.775,64, e o Gtpc-575, com TCO R\$ 9.231,97, pois ambos atendem aos requisitos e possuem os menores custos de propriedade total. Os pesquisadores orientaram os gestores a adquirirem o refrigerador Gtpc-575, pois, mesmo com um TCO maior do que o Gptu - 230pr, ele possui uma capacidade de armazenamento 2,5 vezes maior. Com isso, cumpre-se a quinta etapa.

\section{CONSIDERAÇÕES FINAIS}

Por meio do presente estudo, foi possível verificar que a análise do custo total de propriedade (TCO) pode representar um fator estratégico de grande relevância nas 
Custo Total de Propriedade (TCO) aplicado para a Aquisição de um Refrigerador em uma Microempresa

Andrei Moreira Neves, Daiane Izabele Ferreira de Souza, Moacir Francisco Deimling

empresas. Mediante esse método, as tomadas de decisões são mais assertivas, e os gestores podem aplicar, de forma mais responsável, os recursos financeiros da empresa.

Os itens que apresentam o menor valor para aquisição podem oferecer os maiores custos totais para a organização. Ao longo dos anos, esse valor pode constituir um dos fatores que levam uma empresa à diminuição de seus lucros.

A situação analisada neste trabalho demonstra que é possível realizar uma aquisição que proporcione resultados mais satisfatórios para uma empresa. Embora o valor possa parecer algo sem relevância, considerando-se todas as aquisições necessárias para o funcionamento de uma organização, percebe-se a importância de um processo de TCO. Se todos os custos não forem analisados, a empresa poderá ter uma boa parcela de seus recursos perdidos.

Como sugestão para estudos futuros, sugere-se aplicar essa ferramenta em outras empresas, inseridas em outras atividades econômicas, e também possibilitar uma análise em equipamentos maiores. É possível observar a relevância de um estudo aplicado como este para os pequenos negócios, pois uma decisão errada pode resultar em falência.

\section{REFERÊNCIAS}

Alves, F. J., \& Cardoso, R. L. COQ e TCO: o impacto das decisões de qualidade no custo total de propriedade. FEA-USP. Working Paper. Recuperado em 01 novembro, 2017 , 2017, $\quad$ de <http://www.faf.uerj.br/pastas_prof/ricardo/industrial1/COQ_e_TCO.pdf>.

Blog Algar Telecom. Recuperado em 07 novembro, 2017, de $<$ https://blog.algartelecom.com.br/financas/saiba-o-que-e-o-custo-total-depropriedade-tco-e-sua-importancia-para-o-mundo-dos-negocios/>.

Borinelli, M. L. (2003). Análise de custos de consumidores. (Monografia apresentada ao curso de Gestão Estratégica de Custos). Faculdade de Economia, Administração e Ciências Contábeis, Universidade de São Paulo, FEA/USP, São Paulo. 
Custo Total de Propriedade (TCO) aplicado para a Aquisição de um Refrigerador em uma

Microempresa

Andrei Moreira Neves, Daiane Izabele Ferreira de Souza, Moacir Francisco Deimling

Camargo, T. F., Wernke, R., Zanin, A., \& Scheren, G. (2016). Custo Total de Propriedade: Estudo de Caso em Caminhão (Raçãozeiro) Utilizado na Cadeia de Suprimentosda Suinocultura, Anais do Congresso de Contabilidade da UFRGS, Porto Alegre, RS, Brasil, 01.

Coser, T., \& Souza, M. A. (2017). Custo Total de Uso e Propriedade (TCO): Estudo de Caso em uma Indústria Gráfica do Rio Grande do Sul (RS). Contabilidade Vista \& Revista, 28(1), 67-88.

Cruz, C. Saiba o que é custo total de propriedade (TCO) e sua importância para o mundo dos negócios. Blog Algar Telecom. Recuperado em 07 novembro, 2017, de $<$ https://blog.algartelecom.com.br/financas/saiba-o-que-e-o-custo-total-depropriedade-tco-e-sua-importancia-para-o-mundo-dos-negocios/>.

Ecycle. Recuperado em 09 novembro, 2017, de <https://www.ecycle.com.br/>.

Ellram, L. M. (2002). Total Cost Modeling in Purchasing. CAPS.

Gasparetto, V., \& Silva, I. S. T. da (2004). Custo total de propriedade (TCO): uma ferramenta auxiliar na gestão de custos. Anais do Congresso Brasileiro de Custos. Porto Seguro, BA, Brasil, 11.

Gerhardt, T. E., Silveira, D. T. (organizadores). (2009). Métodos de Pesquisa. Coordenado pela Universidade Aberta do Brasil - UAB/UFRGS e SEAD/UFRGS. Porto Alegre: Editora da UFRGS.

Gil, A. C. (2002). Como elaborar projetos de pesquisa. (4a ed.). São Paulo: Atlas.

Gil, A. C. (2010). Como elaborar projetos de pesquisa. (5a ed.). São Paulo: Atlas.

Godoy, A. S. (1995, maio/junho). Introdução à pesquisa qualitativa e suas possibilidades. RAE - Revista de Administração de Empresas, São Paulo, 35(2), 57 63

M\&M Assessoria Contábil. Recuperado em 09 novembro, 2017, de $<$ https://www.mmcontabilidade.com.br/flash/taxasdepreciacao.html>.

Microcity. Recuperado em 20 novembro, 2017, de <http://www.microcity.com.br/tco-asvantagens-do-total-cost-of-ownership-para-as-empresas>.

Soutes, D. (2007). Custo Total de Propriedade (TCO): É importante? Para quem? Ciências Sociais Aplicadas em Revista (online), 7(3), 83-105. 
Custo Total de Propriedade (TCO) aplicado para a Aquisição de um Refrigerador em uma Microempresa

Andrei Moreira Neves, Daiane Izabele Ferreira de Souza, Moacir Francisco Deimling

Data de Submissão: 22/01/2019

Data de Aceite: 13/04/2020 